\title{
Operational modal analysis on a pump operating at varying speeds: an industrial history case
}

\author{
Vu Viet-Hung ${ }^{1}$, Badri Bechir ${ }^{1}$, Thomas Marc $^{1, a}$ and Jean Pierre ${ }^{2}$ \\ 1 École de technologie supérieure, Montréal, Qc, H3C 1K3, Canada \\ 2 Ville de Montréal - Station d'épuration, Montréal, Qc, H1C 1V3, Canada
}

Received 3 April 2015, Accepted 18 June 2015

\begin{abstract}
Industrial hydraulic pumps are complex structures on which vibrations can be harmful for reliability, fatigue of components and productivity. The application of vibration analysis and diagnosis on a huge water treatment pump at the City of Montreal's facility is presented in this paper. By using a Short Time Auto-Regressive (STAR) method combined with a modal test, it is seen that the dynamic properties and behavior of the pump included both rotating and fixed structures can be evaluated from the experimental analysis on the vibration measurement signal during its normal working condition. Natural frequencies, damping ratios and rotating speed can be clearly identified and monitored. It is shown that the analysis and diagnosis from operational vibration measurement is a very effective technique for the assessment of the huge machines in real working condition.
\end{abstract}

Key words: Hydraulic pump / operational modal analysis / vibration diagnosis / short-time autoregressive

\section{Nomenclature}

\begin{tabular}{|ll|}
\hline $\mathbf{A}_{i}$ & $\begin{array}{l}\text { Matrix of parameters relating } \\
\text { the output } \mathbf{y}(t-i) \text { to } \mathbf{y}(t)\end{array}$ \\
$d$ & Vector dimension or number of sensors \\
$\mathbf{e}(t)$ & Residual vector of all output channels \\
$p$ & Model order \\
$t$ & Time index \\
$T_{\mathrm{s}}$ & Sampling period \\
$\mathbf{y}(t-i)$ & Output vector with time delay $i \times T_{\mathrm{s}}$ \\
$\mathbf{z}(t)$ & Regressor for the output vector $\mathbf{y}(t)$ \\
$\mathbf{\Lambda}$ & Model parameters matrix \\
\hline
\end{tabular}

\section{Introduction}

Vibrations are detrimental for industrial machines and lack of reliability may come from these vibrations during the machine operation. In order to characterise the dynamic behaviour of the structures, modal analysis in nonoperational conditions is a basic technique $[1,2]$. However, due to nonlinear behaviors of dynamic systems, modal analysis in the operational conditions of machines became a necessity to provide a better characterization, analysis and diagnosis in the real life broadband excitations, especially on huge dimension machines where the usual modal

\footnotetext{
${ }^{a}$ Corresponding author: marc.thomas@etsmtl.ca
}

testing suffers lack of applicability [3]. In this paper, this so called operational modal analysis is applied for the vibration analysis and diagnosis of a hydraulic pump in its normal operation [4]. A Short Time Auto-Regressive method (STAR) is used to identify and track the modal parameters of the machines during the operational vibrations. By analyzing the transient vibration signal from fixed and varying operational speeds, the analysis and diagnosis are able to identify the modal parameters and to discriminate the natural frequencies from the resonance frequencies.

\section{The ebara hydraulic pump}

A series of the EBARA pumps (Fig. 1) with three blades and operating at varying speeds is installed at the Montreal city's water treatment plant since the 1970's years. High amplitudes vibrations were witnessed on those pumps at operational speed near 350 RPM [5], and vibration analysis was called in order to identify those high amplitude vibration causes and by consequence to propose corrections.

\section{Short-time autoregressive (STAR) model}

Time series models such as the autoregressive (AR) or autoregressive moving average (ARMA) have been 


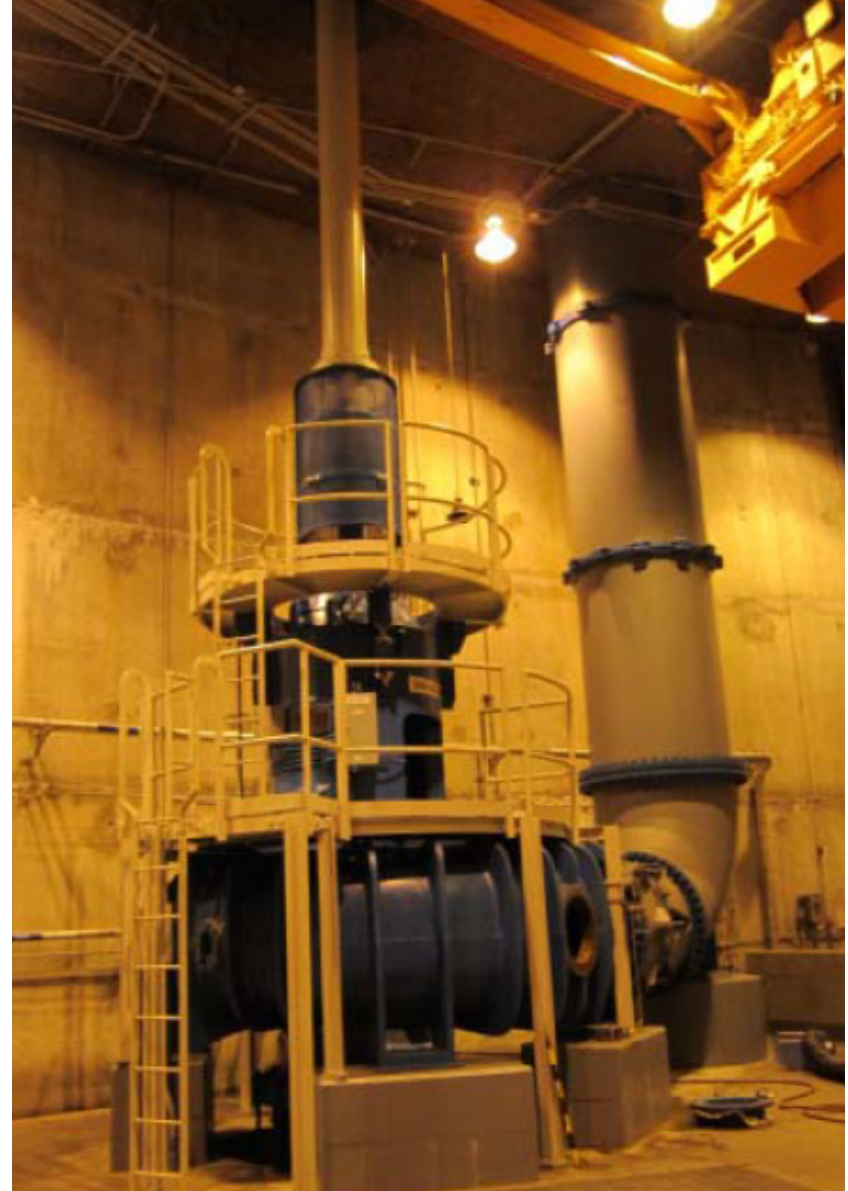

Fig. 1. EBARA pump.

recently used for modal analysis on various applications. As the excitation is generally unknown in operational modal analysis, an AR model at higher order is equivalent to the ARMA model and can be used for the modeling with simple and rapid estimation algorithm. The STAR model has been developed for the operational modal analysis of non-stationary structures and rotating machines [4]. The core mathematic model in the STAR method is the vector-autoregressive (VAR) model where signals from multi channels measurements can be modelled as follows [4]:

$$
\mathbf{y}(t)=\mathbf{\Lambda} \mathbf{z}(t)+\mathbf{e}(t)
$$

where:

$\boldsymbol{\Lambda}=\left[-\mathbf{A}_{1}-\mathbf{A}_{2} \ldots-\mathbf{A}_{p}\right]$ size $d \times d p$ is the parameter matrix;

$\mathbf{A}_{i}($ size $d \times d)$ is the matrix of parameters relating the output $\mathbf{y}(t-i)$ to $\mathbf{y}(t)$;

$\mathbf{z}(t)=\left[\mathbf{y}(t-1)^{\mathrm{T}} \mathbf{y}(t-2)^{\mathrm{T}} \ldots \mathbf{y}(t-p)^{\mathrm{T}}\right]^{\mathrm{T}} \quad($ size $d p \times 1)$ is the regressor for the output vector $\mathbf{y}(t)$;

$\mathbf{y}(t-i)($ size $d \times 1 ; i=1: p)$ is the output vector with delay time $i \times T_{\mathrm{S}}$;

$\mathbf{e}(t)($ size $d \times 1)$ is the residual vector of all output channels considered as the error of model.
The model is estimated by the least squares via the construction of the QR factorization [6] where detail about the signal to noise based model order selection method was also addressed. By applying the VAR model with a sliding window technique as shown in Figure 2, the STAR evolves in the time domain.

As the operation of the machines can deal with time varying effects such as loading, rotation speed or even damages, the operational vibrations usually come from non-stationary sources. To analyze the operational vibration signals, it is therefore beneficial to use the short time window which can be progressed successively in the time domain. In each window, the VAR model is considered stationary. The least squares solution is fast, stable and well-conditioned. Modal parameters are subsequently computed with sufficient accuracy. As the window moves forward, the method is able to track the non-stationary effect. Moreover, the updating of the VAR on model order directly from window to window allows the method for tracking quickly the changes on the system [7]. The advantages of the method are its ability to work fast, accurately online on multi channels measurements, the automation on modes and harmonics distinction [8], its ability to deal with non-stationary signals and with modal parameters uncertainty [9].

\section{Analysis and diagnosis}

\subsection{Measurements at operational fixed speeds}

The vibration measurement is first done on the pump at the constant speed of 360 RPM. Figure 3 shows two measurement positions on three directions RH: Radial Horizontal, RV: Radial Vertical, and RX: Radial Axial [10]. Figure 4 shows the time accelerations (in g) measured in three directions at two points A and B. At a glance, it is observed that the signal can be seen stationary and the vibrations exhibit not much difference in term of amplitudes between the two locations and in the three directions.

In order to compare the vibration levels with standards which are expressed in velocity, Figure 5 shows the velocity spectrum at low frequencies. The rotational frequency is $6 \mathrm{~Hz}$. The main amplitudes are observed at the Blade Pass Frequency (BPF) (18 Hz: 1.5 to $2.0 \mathrm{~mm} . \mathrm{s}^{-1}$ ) and at the fourth harmonic $\left(24 \mathrm{~Hz}\right.$ : amplitude $\left.3 \mathrm{~mm} . \mathrm{s}^{-1}\right)$. A double frequency is observed at the $3 \mathrm{rd}$ harmonic, mostly at the point B. Since the pump is running in steady regime, the double frequency can be explained by a slight asymmetry on the pump structure.

By applying the VAR model presented above to the signal, we can on the other hand construct a stabilization diagram up to model order 50 for both points A and B. The stabilization diagram shows the natural frequencies identified at a range of model orders where the stable frequencies have more chance to be the natural frequencies of the machine. It is seen in Figure 6 the rotating frequency around $6 \mathrm{~Hz}$ which is only stable from model order 40 . Two stable frequencies around $18 \mathrm{~Hz}$ and $24 \mathrm{~Hz}$ match 


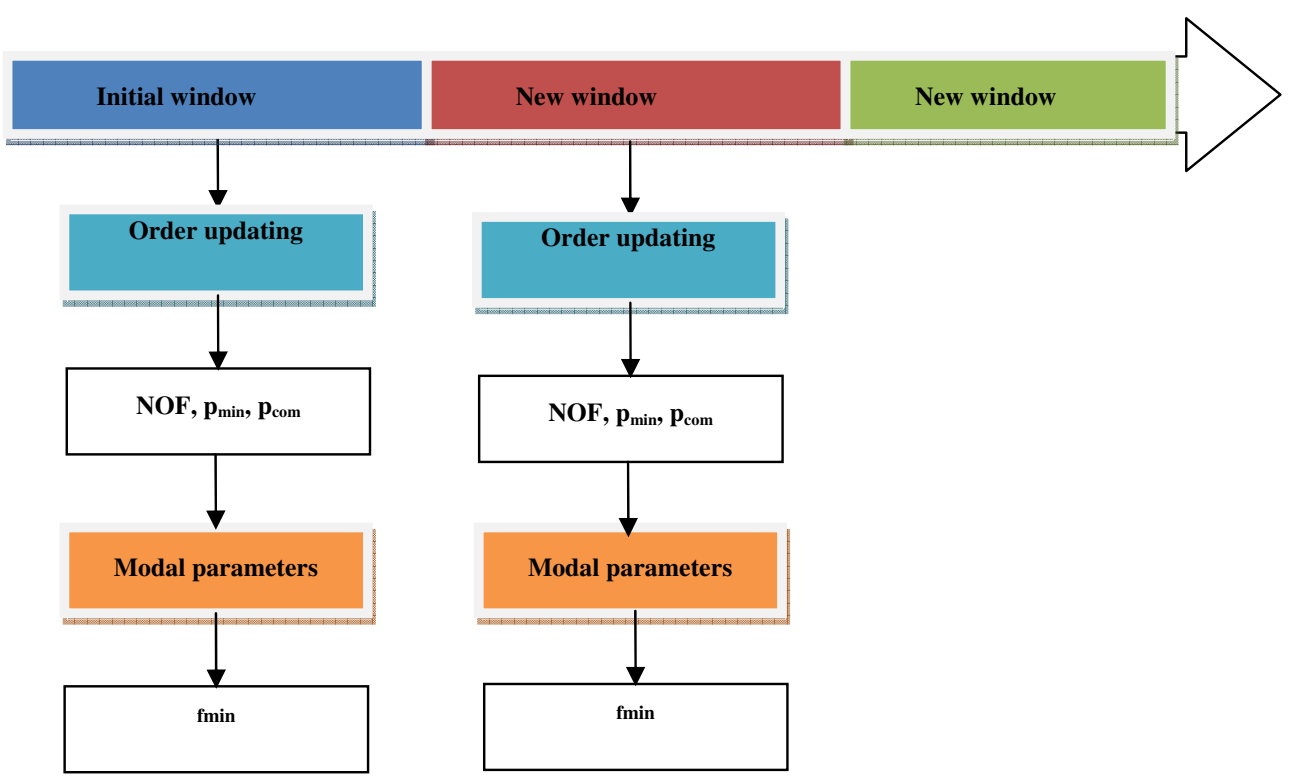

Fig. 2. STAR working scheme.

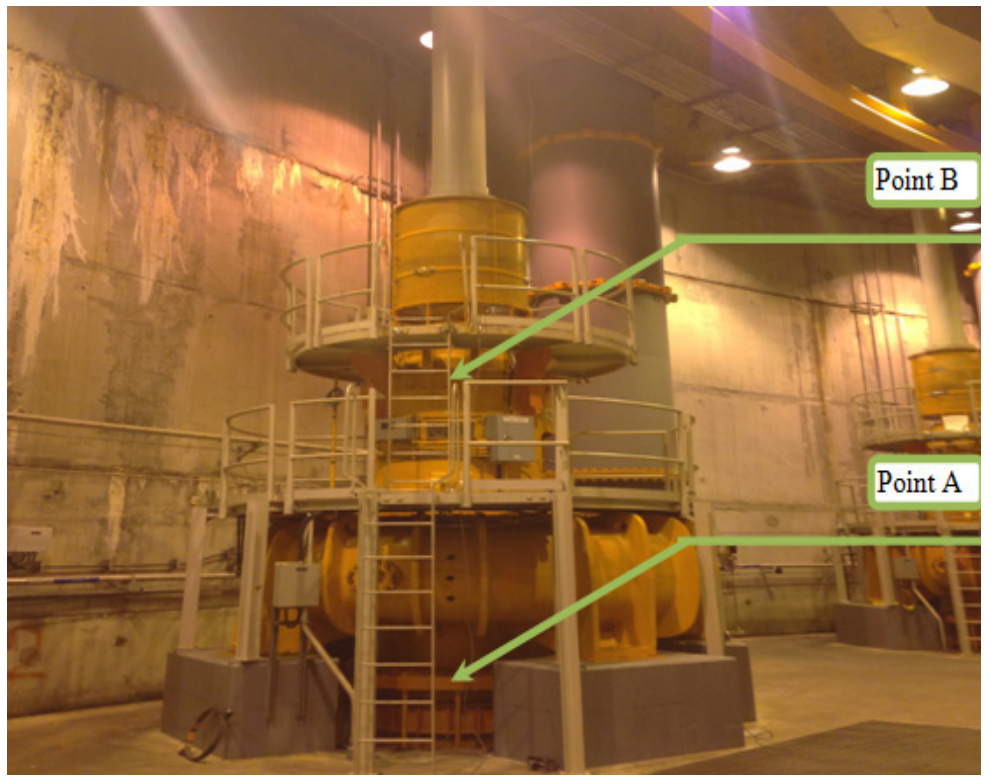

Fig. 3. Measurement locations.

well with the spectra given in Figure 5. However, the discrimination of the natural frequencies and the higher harmonics can be only clarified by the transient testing in the next section.

The RMS velocity (mm.s ${ }^{-1}$ ), accelerations in RMS (mg) and Kurtosis are calculated in Table 1.

The RMS velocity may be compared to the ISO guide: Mechanical vibration - Evaluation of machine vibration by measurements on non-rotating parts - Part 7: Rotodynamic pumps for industrial applications, including measurements on rotating shafts (ISO 10816-7:2009) [11], as shown in Table 2.

By comparing the results, it is possible to say that the vibrations are on the limit of category C- Restricted long term operation. The Kurtosis may be considered as high (greater than 3) [12]. With the data originally sampled at $51200 \mathrm{~Hz}$, Figure 7 shows the acceleration spectrum at high frequencies.

The vibration energy is spread mainly from $1500 \mathrm{~Hz}$ to $4500 \mathrm{~Hz}$ which may be caused by the presence of cavitation. However, the global effective level stays small (max is less than $400 \mathrm{mg}$ ), so the phenomena may be considered as acceptable.

\subsection{Transient testing}

A transient test by varying the speed had been done on the same pump and the same accelerometers locations. 


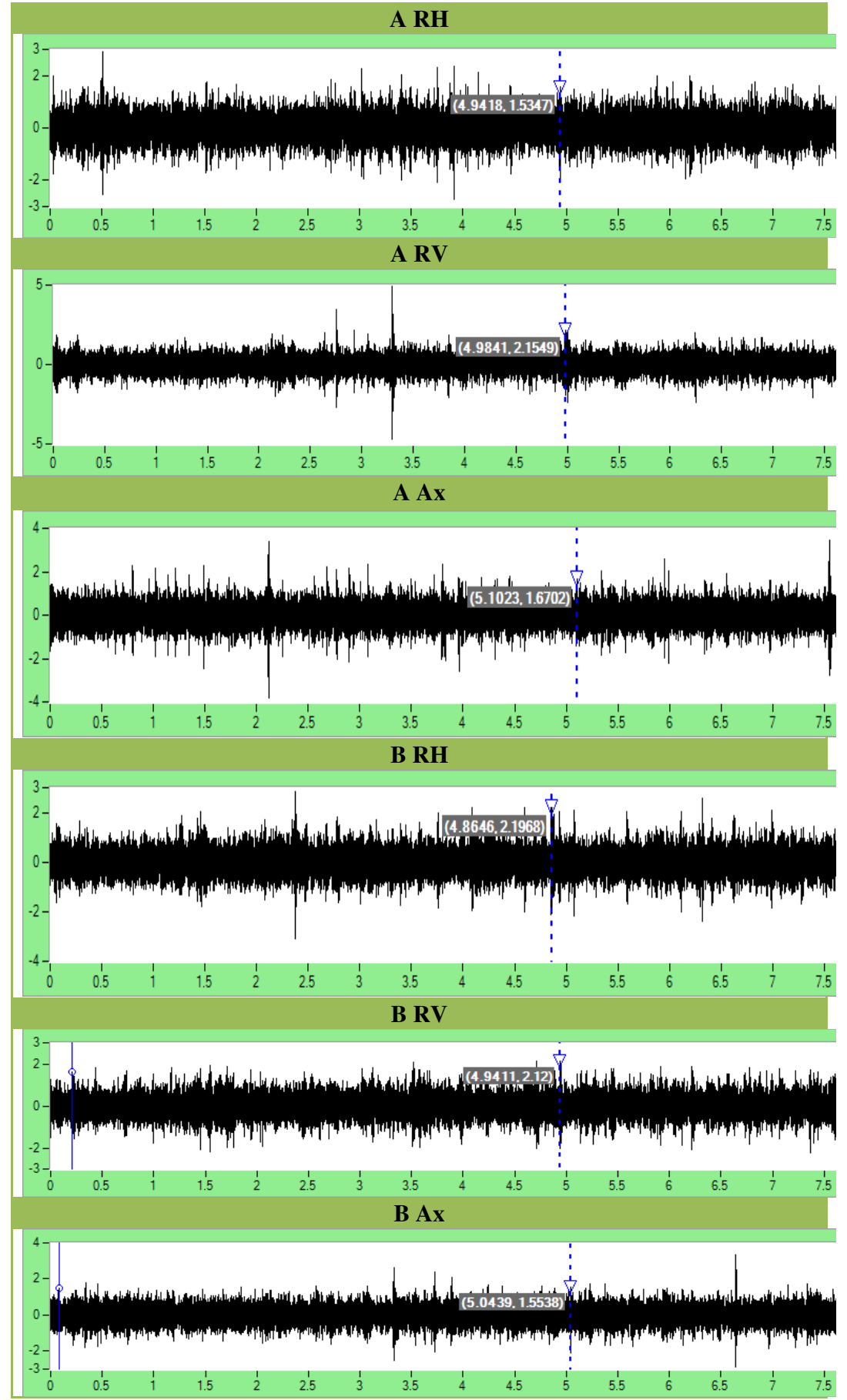

Fig. 4. Acceleration responses.

Table 1. Vibration indicators.

\begin{tabular}{cccccc}
\hline Machine & Point & Dir & RMS Velocity $\left(\mathrm{mm} \cdot \mathrm{s}^{-1}\right)$ & RMS $(\mathrm{mg})$ & KU \\
\hline & & RH & 5.239 & 380 & 3.741 \\
& A & RV & 4.54 & 330 & 5.14 \\
& Ax & 4.27 & 305 & 3.386 \\
GMP 10-1 & & RH & 4.699 & 375 & 3.919 \\
& \multirow{2}{*}{ B } & RV & 3.996 & 351 & 3.678 \\
& & Ax & 5.303 & 427 & 4.413 \\
\hline
\end{tabular}


Vu Viet-Hung et al.: Mechanics \& Industry 17, 101 (2016)
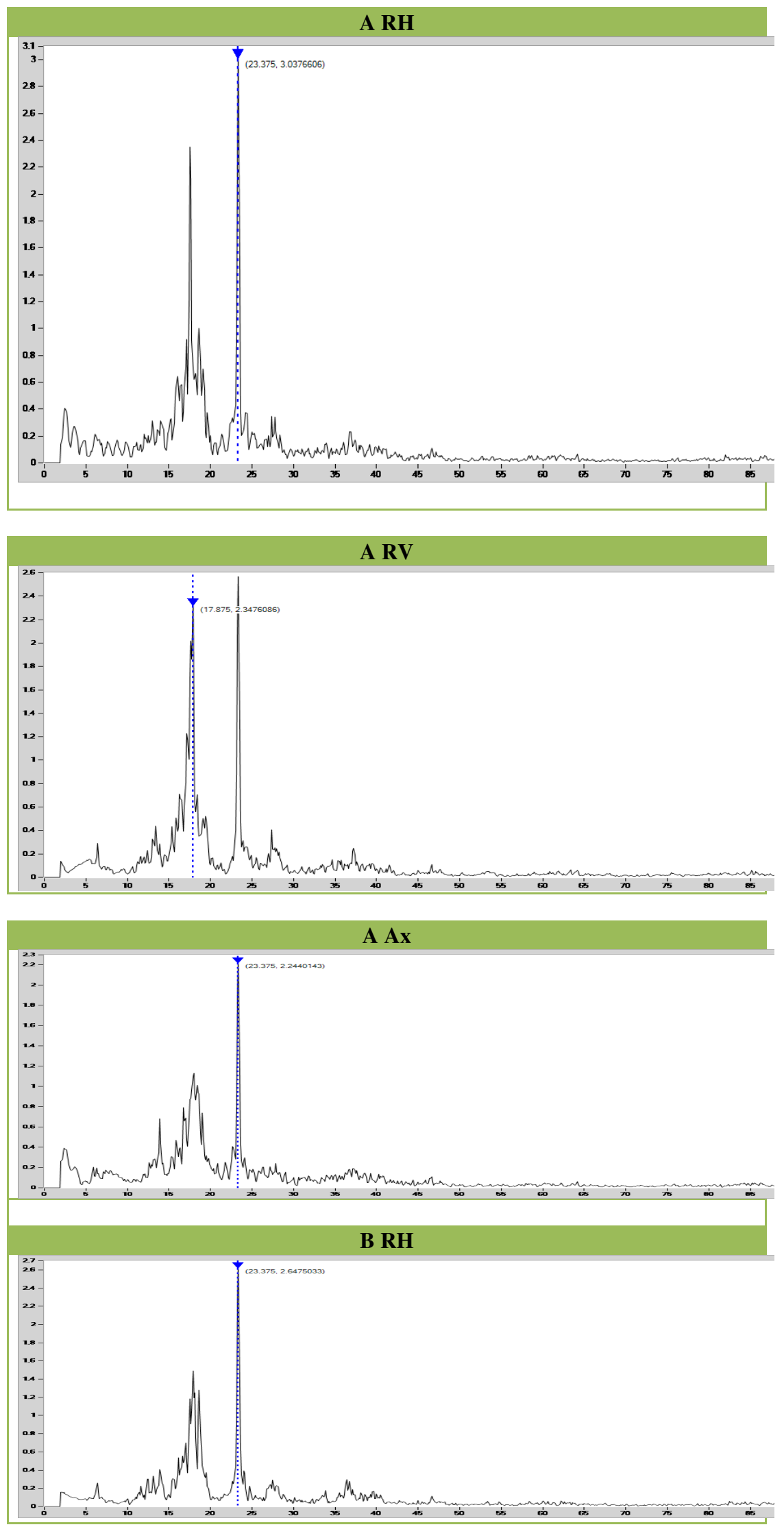

Fig. 5. Velocity spectrum. 

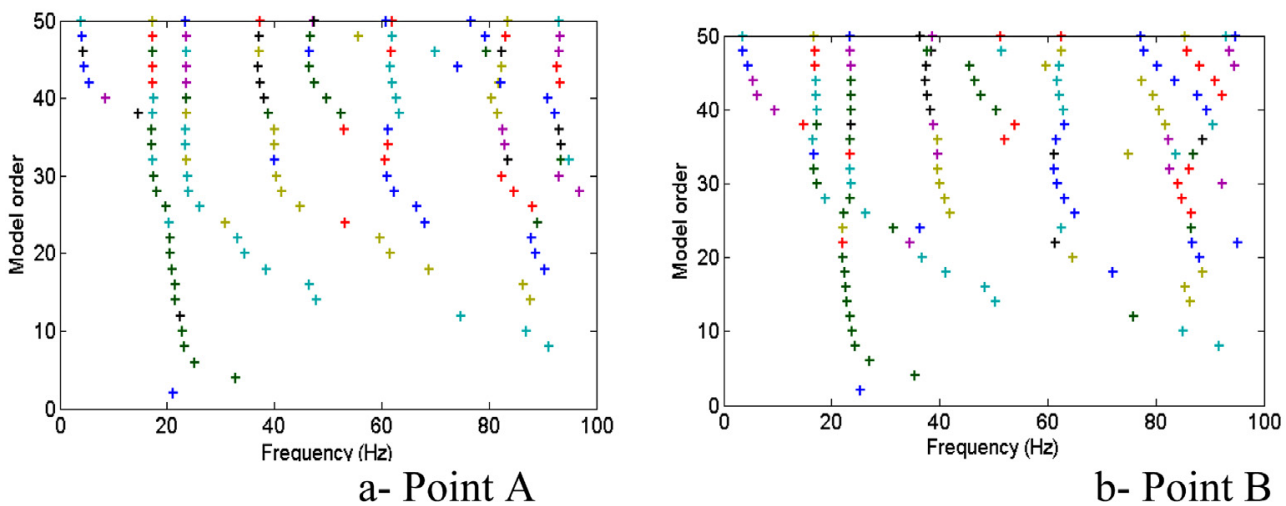

Fig. 6. Stabilization diagram

Table 2. ISO 10816-7:2009.

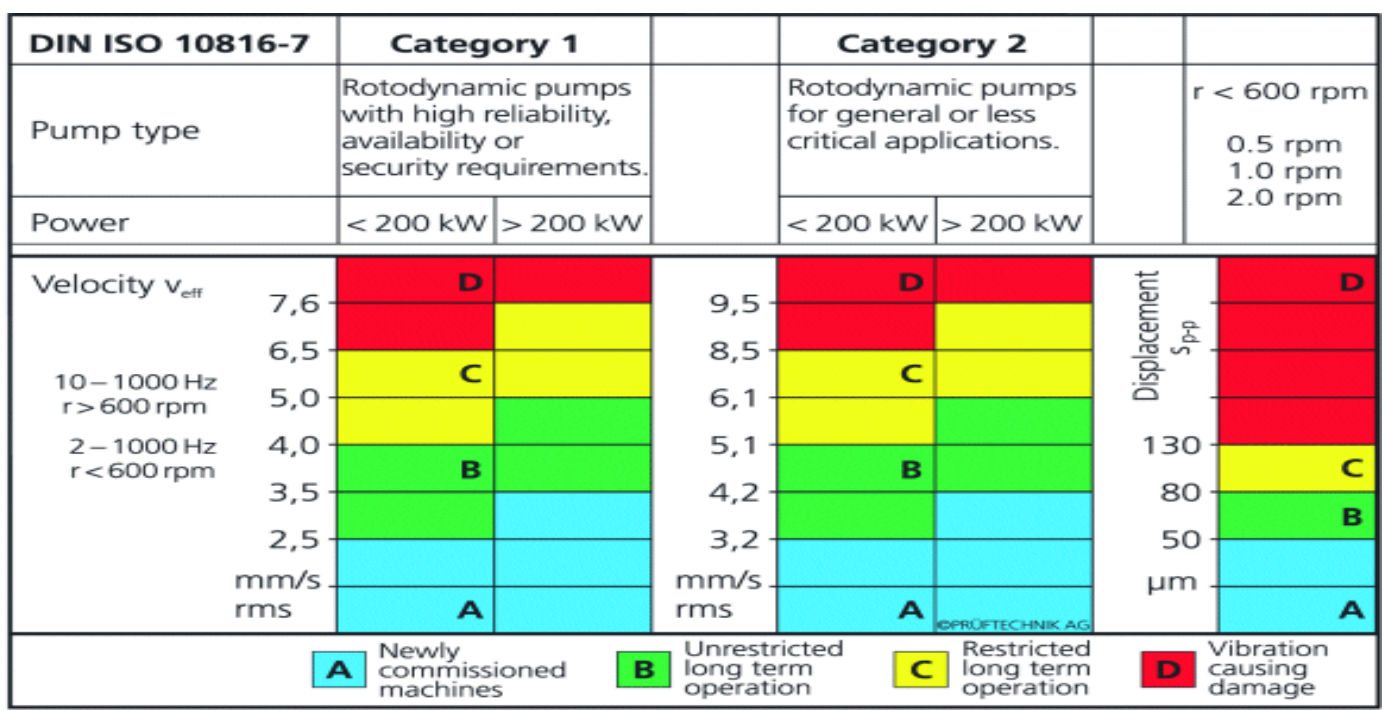

The pump was set to run at the varying speeds from 385 RPM to 340 RPM during $45 \mathrm{~s}$, and then stay at constant speed 340 RPM on the last 45 s. For structural modal analysis purpose, the data have been resampled at the frequency of $512 \mathrm{~Hz}$ (Fig. 8).

Applying the STAR method with a window length $2 \mathrm{~s}$ (1024 samples) and 50\% overlapping, we can see the monitoring of the modal parameters during the non-stationary operation in Figure 9 (frequency monitoring) and Figure 10 (damping ratio monitoring). From the monitoring of the modal parameters obtained from only vibrational responses during the normal operation, it is possible to see that all the basic modes of the structure are present in the vibration spectra. All the modal parameters are stable during the operation when varying speed or at fixed speed, while the harmonic frequencies are varying (4th harmonic at $24 \mathrm{~Hz}$ ). During the first half of the transient loading time, the operation speed varies from 385 RPM to $340 \mathrm{RPM}$ whose the 4 th harmonic is closed to the $2 \mathrm{nd}$ structural mode at $25 \mathrm{~Hz}$.

A resonance occurs during the first half time and the STAR method therefore tracks the varying operational speed with the corresponding damping ratios close to zero. During the 2nd half time, the STAR method identifies and tracks all the structural modes with constant natural frequencies around $18 \mathrm{~Hz}, 25 \mathrm{~Hz}, 38 \mathrm{~Hz}, 50 \mathrm{~Hz}$, $62 \mathrm{~Hz}$ and stable damping ratios around $5 \%$.

The monitoring results from the non-stationary vibration validate also the spectrum constructed from the fixed operational speed in the fixed speed testing section (Fig. 5). It is then well seen that the first structural mode is found around $18 \mathrm{~Hz}$ close to the $\mathrm{BPF}$ (third harmonic) and another resonance occurs near $25 \mathrm{~Hz}$ (close to 4th harmonic).

\section{Preliminary solution proposals}

It is seen from the diagnosis that a structural mode around $17-18 \mathrm{~Hz}$ is found close to the 3rd harmonic and another structural mode around $24 \mathrm{~Hz}$ is found close to the 4 th harmonics. In order to avoid the resonance, a rough numerical model of the pump has been built in Ansys and several preliminary structural modifications are considered on the rotating and the fix parts respectively to modify the natural frequencies of those two structural 
Vu Viet-Hung et al.: Mechanics \& Industry 17, 101 (2016)

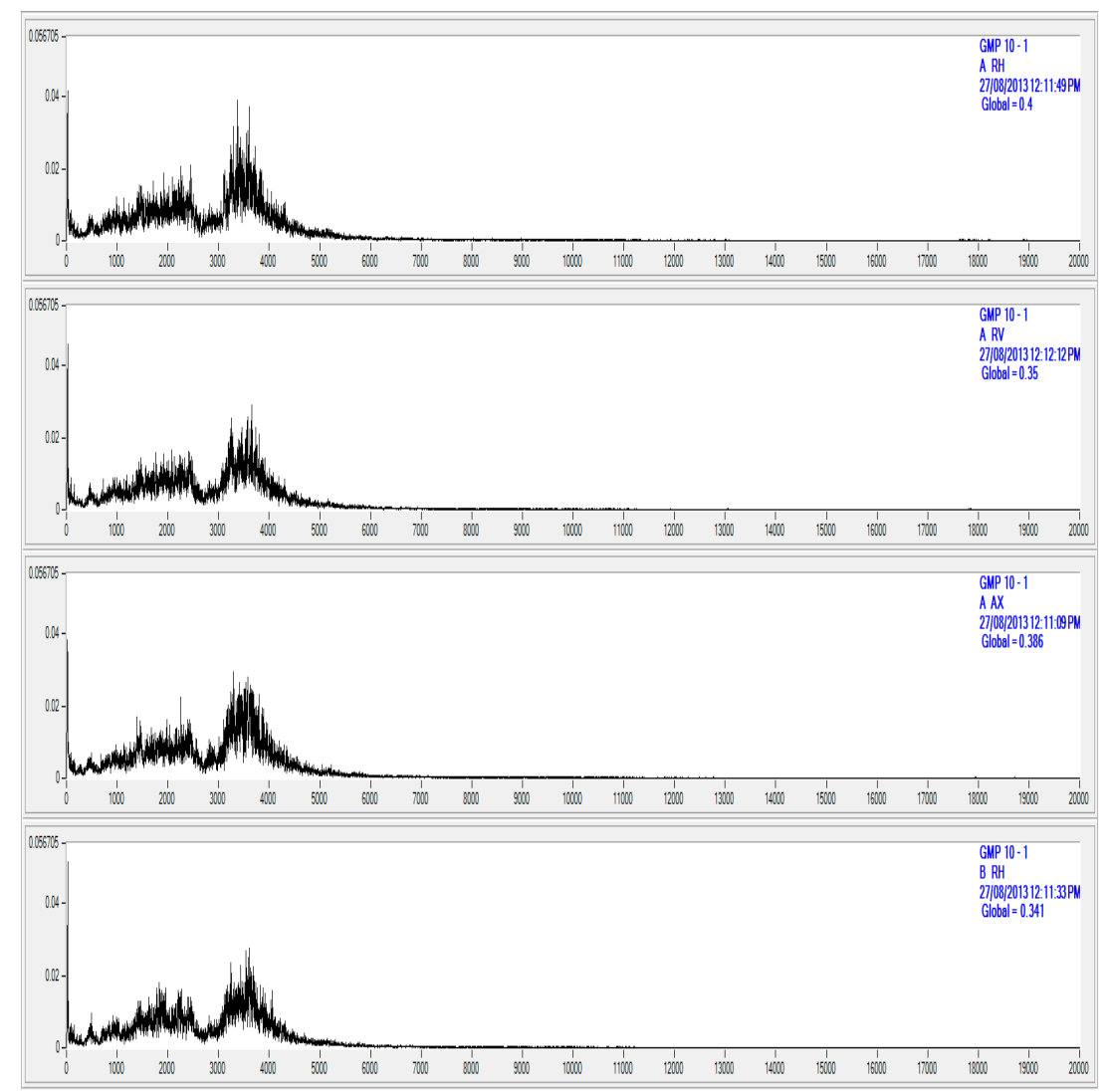

Fig. 7. Acceleration spectrums at high frequencies.

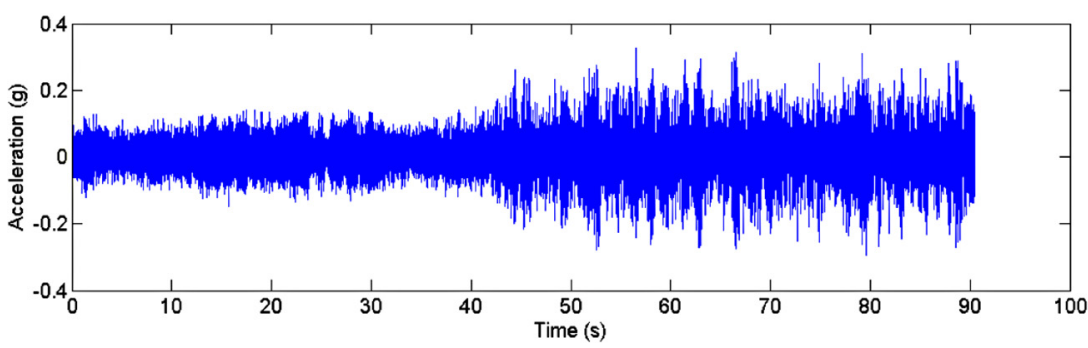

Fig. 8. Transient operational signal, point A.

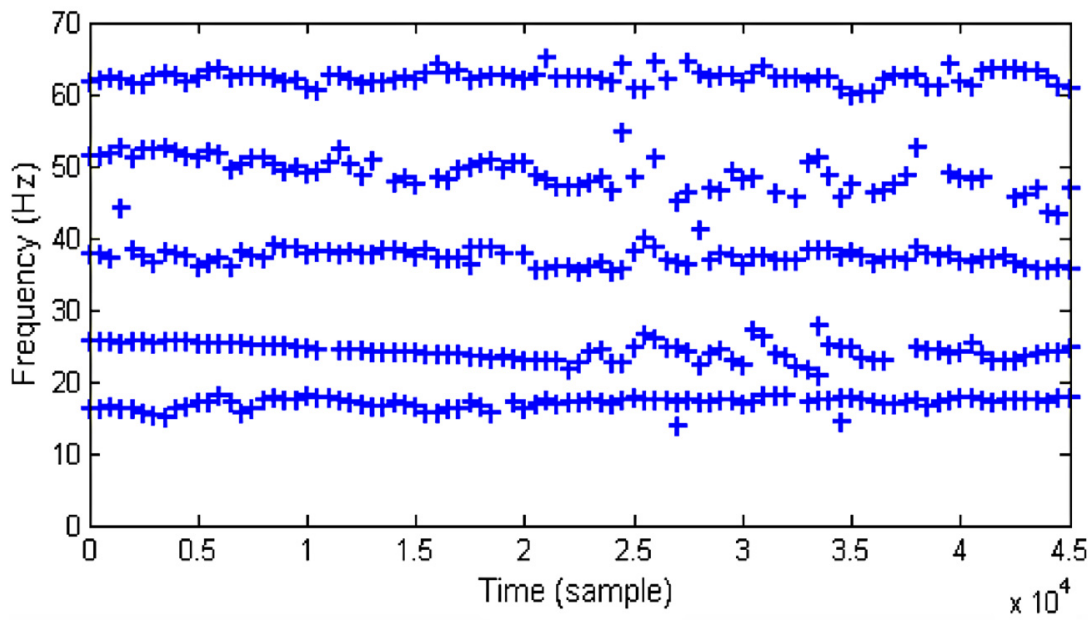

Fig. 9. Frequency monitoring during speed variations. 


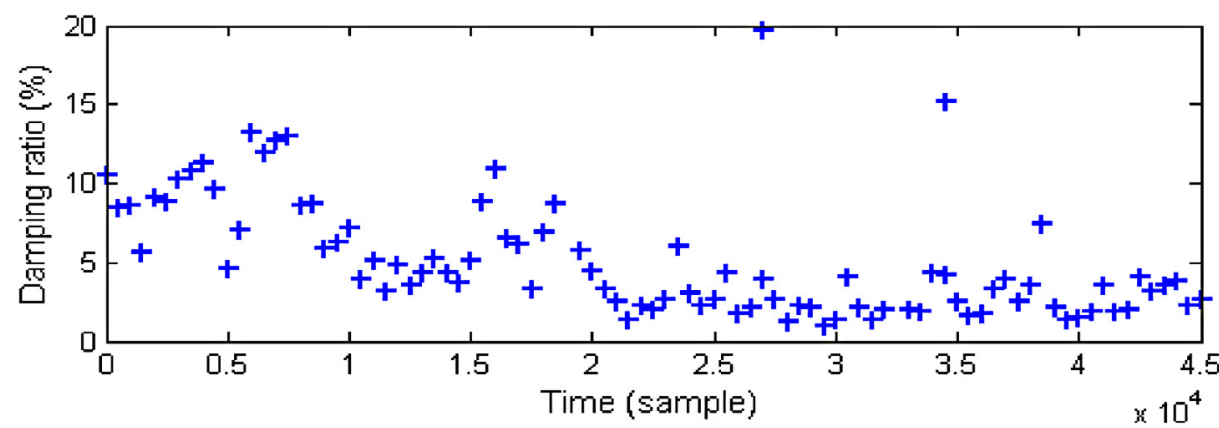

a- Mode $18 \mathrm{~Hz}$

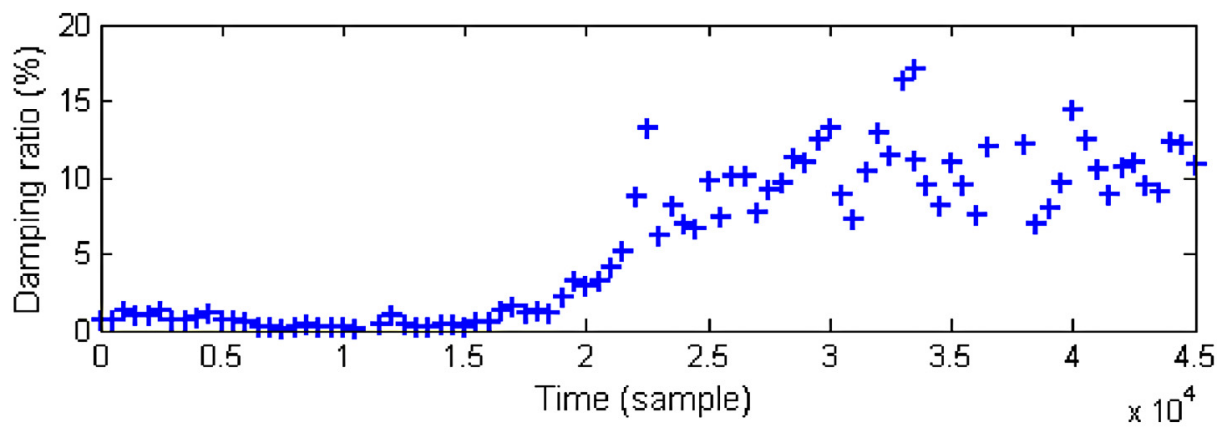

b- Mode $24 \mathrm{~Hz}$

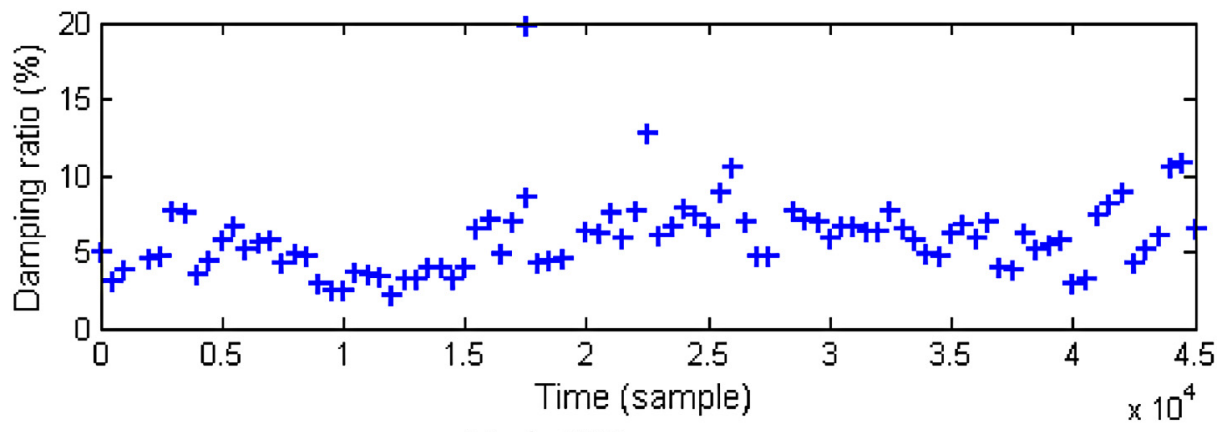

c- Mode $38 \mathrm{~Hz}$

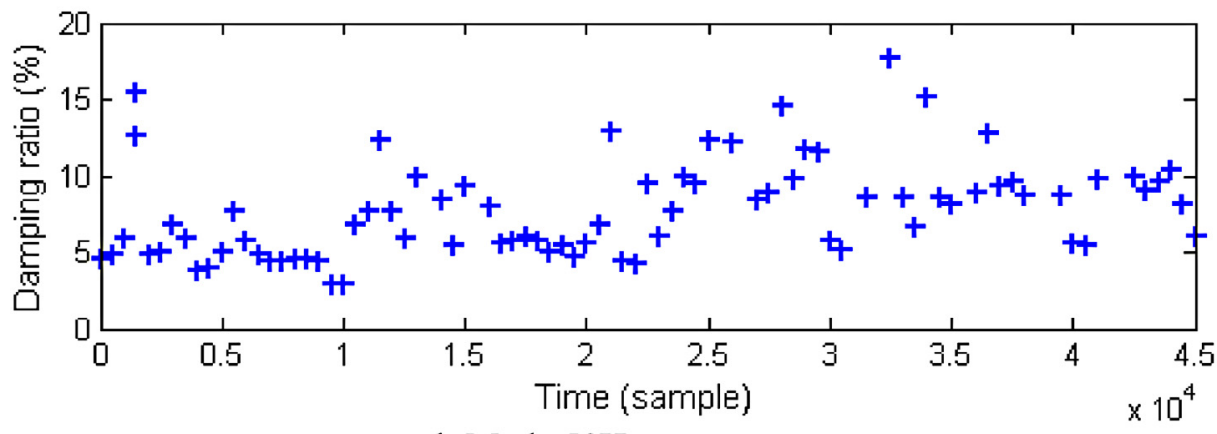

d- Mode $50 \mathrm{~Hz}$

Fig. 10. Damping ratios monitoring during speed variation.

modes. Detail of the modification with the design is ongoing research and is proposed on the part 2 of the project.

\subsection{Creation of an additional support to the shaft}

The shaft is the rotating part of the pump currently has three supports. As the superior part of the pump is the only accessible location for the modification on the shaft, it is proposed to add a 4 th support to the shaft. It is understood that the stiffness of the additional support is limited so several stiffness values are considered. It is found that the linear stiffness of the additional support must be at least $1 \mathrm{E} 8 \mathrm{~N} . \mathrm{m}^{-1}$ to provide a notable change on the natural frequencies. Stiffness at $1 \mathrm{E} 9 \mathrm{~N} . \mathrm{m}^{-1}$ is proposed for the simulation. Figure 11 shows the disposition 

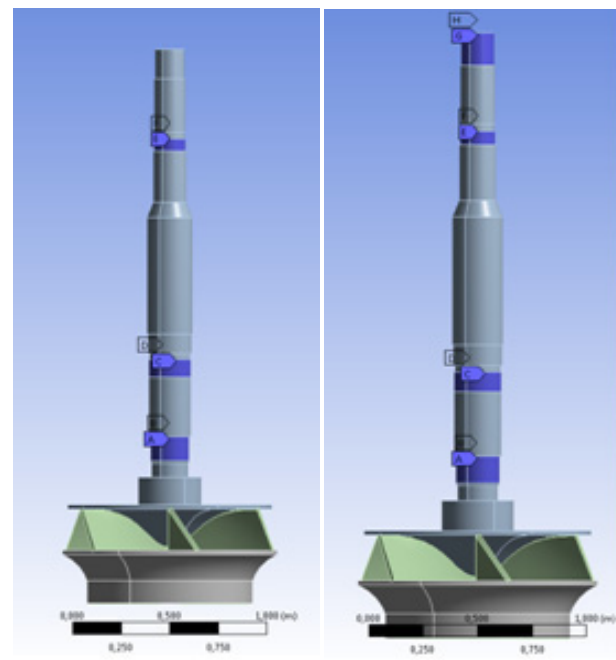

\begin{tabular}{|l|c|l|}
\hline \multirow{2}{*}{ Mode } & \multicolumn{2}{|l|}{ Natural frequencies } \\
\cline { 2 - 3 } & Before & After \\
\hline 1 & 16.53 & 53.00 \\
\hline 2 & 16.64 & 53.21 \\
\hline 3 & 45.23 & 127.11 \\
\hline 4 & 96.95 & 127.35 \\
\hline 5 & 97.54 & 225.57 \\
\hline
\end{tabular}

Fig. 11. Additional support.

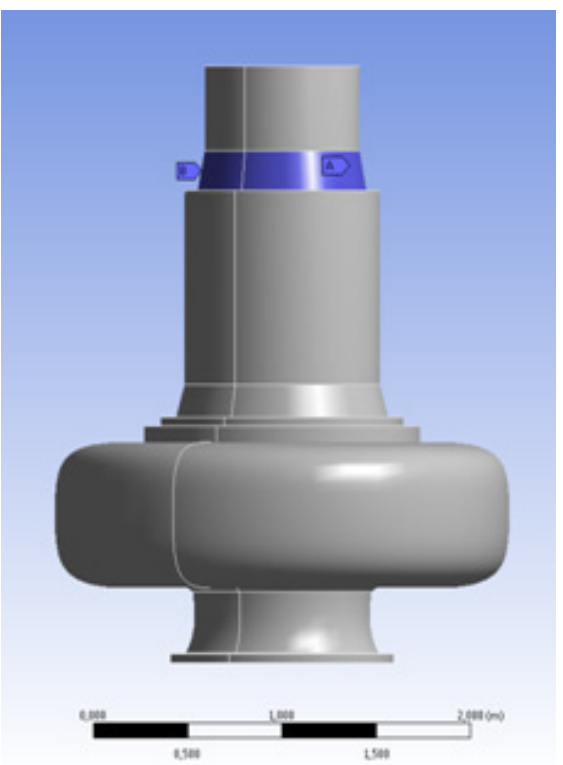

\begin{tabular}{|l|c|c|}
\hline \multirow{2}{*}{ Mode } & \multicolumn{2}{|l|}{ Natural frequencies } \\
\cline { 2 - 3 } & Before & After \\
\hline 1 & 16.7 & 40.2 \\
\hline 2 & 17.7 & 62.0 \\
\hline
\end{tabular}

Fig. 12. Chassis reinforcement.

of the current support and the additional support at the top of the shaft. The model presents the numerical natural frequencies before and after installation of the 4 th support. It is seen that the natural frequencies are significantly displaced and the resonance at the 3rd and 4th harmonics are avoided.

\subsection{Chassis reinforcement}

Another solution is on the fixed part, the chassis of the pump. It is seen that the lower part of the chassis is fixed to the concrete foundation. However the upper part is more flexible. A structural solution can be considered to add the steel beam connecting the chassis to the concrete wall in order to reinforce its stiffness. Figure 12 presents the chassis model with the ring support at the upper part. As similar to the shaft, a linear stiffness at $1 \mathrm{E} 9 \mathrm{~N} . \mathrm{m}^{-1}$ is proposed showing the change on the natural frequencies of the chassis itself. It is seen that the resonance on the chassis is avoided effectively.

\section{Conclusions}

Hydraulic pumps are complex industrial structures where the excessive vibrations can be threats to the structural integrity. Operational Modal Analysis (OMA) 
adapted for non-stationary systems like STAR can be used for vibration analysis and diagnosis on the heavy industrial machines without knowing of the mechanical and geometric properties, neither the excitation. The method can identify and track accurately the modal parameters during the normal operation of the pump at constant or varying speeds. The rotating speed and the resonance can also be revealed during the monitoring. By combining with the amplitudes assessment, the dynamic picture of the machine is clarified and enlightened to the operators. Several preliminary modifications are also proposed to correct the problem.

\section{References}

[1] D.J. Ewins, Modal testing: Theory and practice, Research Studies Press, 2000

[2] N.M.M. Maia, J.M.M. Silva, Modal analysis identification techniques, Royal Society 359-2001 (2001) 9-40

[3] L. Hermans, H. Van der Auweraer, Modal testing and analysis of structures under operational conditions: Industrial applications, Mech. Syst. Signal Process. 13 (1999) 193-216.

[4] V.H. Vu, M. Thomas, A.A. Lakis, L. Marcouiller, Shorttime autoregressive (STAR) modeling for operational modal analysis of non-stationary mechanical systems, Chapter 3 on book: Vibration and structural acoustic analysis, Springer, 2011, pp. 59-77, ISBN: 9789400717022
[5] F. Provost, Rapport d'analyse de vibration, pompe EBARA, Delom Services, 2010, 26 p.

[6] V.H. Vu, M. Thomas, F. Lafleur, L. Marcouiller, Toward an automatic spectral and modal identification from operational modal analysis, J. Sound Vibr. Elsevier 332 (2013) 213-227

[7] V.H. Vu, M. Thomas, A.A. Lakis, L. Marcouiller, Operational modal analysis by updating autoregressive model, Mech. Syst. Signal Process. (MSSP) Elsevie 25 (2011) 1028-1044

[8] V. H. Vu, M. Thomas, F. Lafleur, Harmonic and Modal Frequency Discrimination in Time Domain Operational Modal Analysis, Mechanics \& Industry 15 (2014) 29-37

[9] V.H. Vu, M. Thomas, Uncertainty of modal parameters by operational modal analysis, Mechanics \& Industry 15 (2014) 153-158

[10] B. Badri, V.H. Vu, M. Thomas, Experimental vibration analysis on EBARA pump, Technical Report (in french), ETS, Montreal, 2014, 17 p.

[11] ISO 10816-7, Mechanical vibration - Evaluation of machine vibration by measurements on non-rotating parts Part 7: Rotodynamic pumps for industrial applications, including measurements on rotating shafts, 2009, 17 p.

[12] M. Thomas, Reliability, predictive maintenance and machinery vibration (in french). Presses de l'Université du Québec, 2011, 633 p., D3357, ISBN 978-2-7605-3357-8 